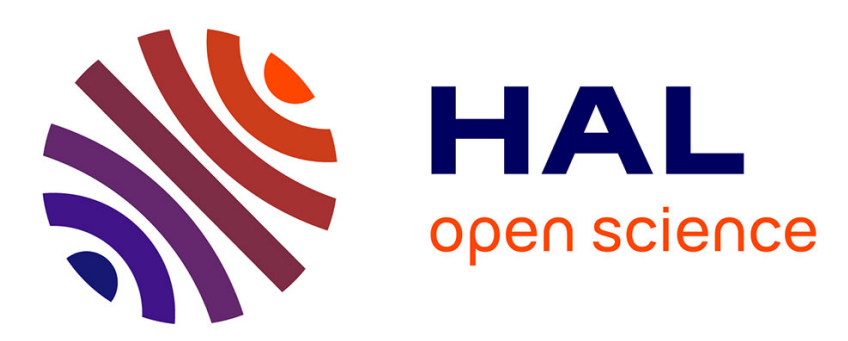

\title{
Thermal conductivity and transport modes in glassy GeTe 4 by first-principles molecular dynamics
} Thuy-Quynh Duong, Carlo Massobrio, Guido Ori, Mauro Boero, Évelyne Martin

\section{To cite this version:}

Thuy-Quynh Duong, Carlo Massobrio, Guido Ori, Mauro Boero, Évelyne Martin. Thermal conductivity and transport modes in glassy GeTe 4 by first-principles molecular dynamics. Physical Review Materials, 2019, 3 (10), 10.1103/PhysRevMaterials.3.105401 . hal-02312816

\section{HAL Id: hal-02312816 https://hal.science/hal-02312816}

Submitted on 17 Nov 2020

HAL is a multi-disciplinary open access archive for the deposit and dissemination of scientific research documents, whether they are published or not. The documents may come from teaching and research institutions in France or abroad, or from public or private research centers.
L'archive ouverte pluridisciplinaire HAL, est destinée au dépôt et à la diffusion de documents scientifiques de niveau recherche, publiés ou non, émanant des établissements d'enseignement et de recherche français ou étrangers, des laboratoires publics ou privés. 


\title{
Thermal conductivity and transport modes in glassy $\mathrm{GeTe}_{4}$ by first-principles molecular dynamics
}

\author{
Thuy-Quynh DUONG \\ Univ. Lille, CNRS, Centrale Lille, \\ ISEN, Univ. Valenciennes, \\ UMR 8520 - IEMN, F-59000 Lille, France
Carlo MASSOBRIO, Guido ORI, Mauro BOERO Université de Strasbourg, CNRS, UMR 7504, Strasbourg F-67034, France. \\ Institut de Physique et Chimie des Matériaux de Strasbourg, \\ Evelyne MARTIN * \\ Univ. Lille, CNRS, Centrale Lille, \\ ISEN, Univ. Valenciennes, \\ UMR 8520 - IEMN, F-59000 Lille, France.
}

\begin{abstract}
First principles molecular dynamics is employed to investigate thermal transport in glassy $\mathrm{GeTe}_{4}$, a subsystem of several ternary phase-change materials. As a first result, we found modes localized on a few atoms in the vibrational density of states. The thermal transport is further rationalized by calculating the thermal conductivity for a range of system sizes and shapes via the approachto-equilibrium methodology. By considering the length dependence of the thermal conductivity, we provide evidence of propagative modes with mean free paths as long as $6 \mathrm{~nm}$, i. e. well beyond short range order distances. Extrapolation of our bulk thermal conductivity to macroscopic sizes is in full agreement with the experimental values. Finally, we assess phenomenological models developed for the thermal conductivity of disordered materials, by enriching their intrinsic significance via the insertion in their analytical expression of values obtained via first principles molecular dynamics.
\end{abstract}

\section{INTRODUCTION}

Materials in a disordered phase have an increasing importance in technology [1]. For instance, glassy chalcogenides are used in next generation non-volatile memories. The principle of such devices is to assign a bit value to the material state, i.e. crystalline or disordered, through phase switching obtained via Joule heating. Thermal management is a major concern when developing phase-change random access memories (PCRAM), in particular to limit the amount of energy needed to induce the phase switching, and avoid wasting a significant part of it or cross-talking to neighboring units [4. Optimizing the thermal design requires an in-depth understanding of the heat transport in different parts/phases of the PCRAM. However, in spite of the strong drive of these technological requirements, heat transport in disordered materials remains elusive.

In principle, the lack of order beyond nearest neighbor distances in disordered materials is not compatible with the notion of phonons. However, in the case of amorphous silicon, both theory [5, 6] and experiments 7] concur that propagative modes do exist and the heat

*evelyne.martin@univ-lille.fr carriers can travel distances as high as micrometers. An additional contribution to thermal conductivity has been ascribed to diffusive modes, i.e. modes clustering in order to transport heat but lacking a well-defined mean free path, or having mean free paths smaller than the nearest neighbor distances. Apart from the case of amorphous silicon, propagative modes have not been identified in other amorphous materials 8]. The question arises on whether this is the case because their mean free paths are under the resolution of experimental probes (i. e. $\approx 5 \mathrm{~nm}$ ) or they are actually absent in any other disordered system. On the theoretical side, evidence of such modes could be collected by calculating the thermal conductivity as a function of the size along the heat transport direction. Nowadays, it is possible to achieve this goal in a quantitative fashion, by relying on firstprinciples molecular dynamics (FPMD) in the framework of density functional theory (DFT). However, given the high computational cost of the Green-Kubo 9 or direct methods 10, FPMD have to be combined with more tractable, alternative approaches. With this purpose in mind, we calculated via FPMD the thermal conductivity of a disordered material (glassy $\mathrm{GeTe}_{4}$ ) [11] by taking advantage of the approach-to-equilibrium molecular dynamics (AEMD) methodology [12. In this first joint application of FPMD and AEMD, we showed that the inherent time intervals are within the reach of FPMD, in 
spite of a substantial temporal relaxation from an out-ofequilibrium situation (phase 2 of AEMD) featuring two adjacent parts of a periodic volume kept at different temperatures (phase 1 of AEMD). The thermal conductivity of glassy $\mathrm{GeTe}_{4}$ was severely underestimated (by a factor of ten), due to the existence of unavoidable size effects in terms of both dimensions parallel and perpendicular to the direction of thermal transport. To substantiate this statement, further calculations were carried out in Ref. 13. by duplicating the cell dimensions in the direction of thermal transport (from $N=185$ to $N=370$ atoms). We found an increase of the thermal conductivities by a factor of three. Despite these instructive indications, the quest for a precise understanding of thermal transport in glassy $\mathrm{GeTe}_{4}$ calls for further calculations and analyses. One has to account for larger sizes and various geometries along (longitudinally) and across (transversally) the direction of the heat flux. Moreover, it is desirable to elucidate the mechanism of thermal transport in terms of vibrational properties.

Here we achieve these goals by calculating the thermal conductivity of $\mathrm{g}-\mathrm{GeTe}_{4}$ for four systems differing by the simulation box dimensions (see Fig. 1). We are able to extract the asymptotic behavior of the thermal conductivity as a function of system size in the direction of thermal transport and gather information on the sensitivity to the transversal extension of the simulation box. In terms of vibrational properties, we infer the existence of localized and extended modes.

The present paper is organized as follows. In section II we provide details on the computational methodology and present the atomic models of the $\mathrm{GeTe}_{4}$ glass that we characterize from the structural and vibrational point of view. Section III A focuses on the vibrational properties, while section IIIB is devoted to the calculation of the thermal conductivity. In section IV phenomenological models for heat transport are revisited in light of the results obtained from our FPMD calculations, thereby allowing to assess their predictive power. Conclusions are contained in sections $\mathrm{V}$

\section{FPMD METHODOLOGY AND MODEL CONSTRUCTION}

First-principles molecular dynamics simulations were carried out using the Car-Parrinello 14 method as implemented in the CPMD code 42. In the DFT framework, we used the exchange functional of Becke 15] and the correlation part of Lee, Yang and Parr 16] (BLYP). FPMD results on glassy g-GeTe ${ }_{4}$ made available in a previous work highlighted the better performances of BLYP when compared to PBE [17] in terms of agreement with available experimental data. For these reasons, the present FPMD study relies on BLYP for the production of the temporal trajectory exploited to convey information on the thermal properties. Other relevant technical details go as follows. Core-valence interactions are described via norm-conserving pseudo-potential as devised according to the prescription of Troullier and Martins [18. A planewave basis set is chosen for the representation of the valence electrons with an energy cutoff of 50 Ry. The Brillouin zone integration is restricted to the $\Gamma$ point as legitimate for calculations on extended (and non-metallic) disordered systems. In line with the rationale developed in Ref. 19] we accounted for dispersion forces by adopting the Grimme-D2 formula proposed by Grimme [19, 20].

As detailed in Ref. 19, the structure of glassy $\mathrm{GeTe}_{4}$ resulting from this choice (BLYP and the Grimme-D2 dispersion forces) is close to the one pertaining to a chemically ordered network (CON) of tetrahedra. This can be appreciated by comparing the corresponding CON and BLYP-D2 partial coordination numbers (Table I).

TABLE I: Partial coordination numbers.

\begin{tabular}{c|c|c} 
& CON & BLYP-D2 \\
\hline$n_{\mathrm{GeGe}}$ & 0 & 0.37 \\
\hline$n_{\mathrm{GeTe}}$ & 4 & 3.60 \\
\hline$n_{\mathrm{TeGe}}$ & 1 & 0.91 \\
\hline$n_{\mathrm{TeTe}}$ & 1 & 1.40
\end{tabular}

In such arrangements, the large majority of Te atoms can be found in the $\mathrm{GeTe}_{4}$ tetrahedron or in $\mathrm{Te}_{n}$ configurations, a third possibility being the involvement in connections between a given tetrahedron and a $\mathrm{Te}_{n}$ chain. It is useful to point out that BLYP (with or without the Grimme-D2 correction) favors the tetrahedral coordination together with a strong tendency to chemical order, unlike PBE for which $n_{\mathrm{TeTe}}^{\mathrm{PBE}-\mathrm{D} 2}=2.47$ (see Ref. 19).

Our methodology for the calculation of the thermal conductivity relies on an accurate control of the ionic temperature on distinct parts of the system. To this end we employed the Nosé-Hoover thermostat [21, 22 , on the ionic degrees of freedom. For the electronic degrees of freedom, adiabatic conditions are ensured via the Blöchl-Parrinello 23. thermostat. However, this second thermostat was suppressed whenever the ionic thermostat was not activated to avoid unnecessary thermal control of the electronic degrees of freedom. This is, for instance, the case of phase 2 in the AEMD procedure. The integration step is 5 a.u. (0.12 fs) throughout our calculations.

The initial configuration containing $N=215$ atoms, 43 Ge and $172 \mathrm{Te}$, is selected from the BLYP trajectory produced at $T=300 \mathrm{~K}$ in Ref. 19. This first model, hereafter termed $\mathrm{B}$, consists in a cubic supercell of edge equal to $19.24 \AA$. To assess the dependence of our calculated thermal conductivity in terms of system dimensions along and across the direction of thermal transport, model $\mathrm{B}$ was replicated and/or rotated to obtain the three additional systems B2, B2r and B3 shown in Fig. 1.

The characteristics of the 4 models are given in Table II.

For the calculation of the vibrational density, we resorted to a finite difference method implemented in the 


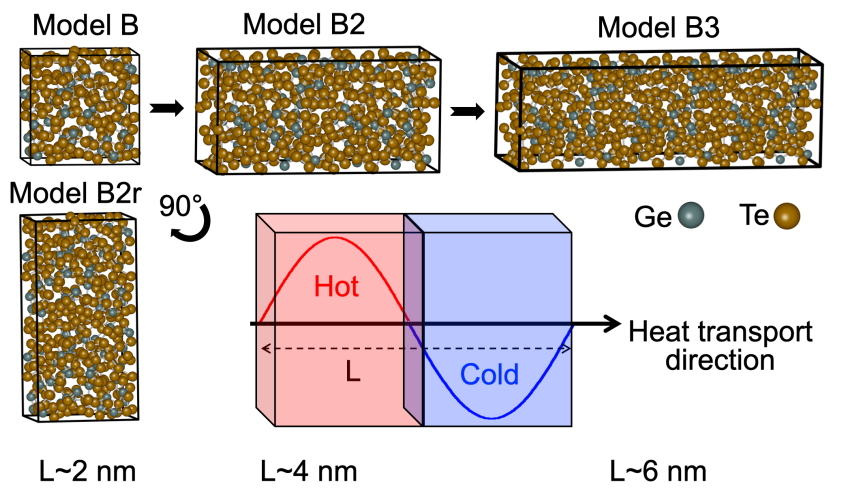

FIG. 1: The four atomic models studied in the present work. $L$ is the cell length in the heat transport direction. The representations were obtained using iRASPA 24].

TABLE II: Characteristics of the 4 models. \begin{tabular}{l|l|l|l|} 
Name & N & Section $\left(\AA^{2}\right)$ & L $(\AA)$ (as in Fig. 1)
\end{tabular}

\begin{tabular}{c|c|c|c}
\hline B & 215 & $19.24 \times 19.24$ & 19.24 \\
\hline B2 & 430 & $19.24 \times 19.24$ & 38.48 \\
\hline B3 & 645 & $19.24 \times 19.24$ & 57.72 \\
\hline B2r & 430 & $19.24 \times 38.48$ & 19.24
\end{tabular}

CPMD code on a set of configurations of the B model.

\section{THERMAL PROPERTIES}

\section{A. Analysis of vibrational modes}

Thermal properties of glassy $\mathrm{GeTe}_{4}$ can be rationalized in terms of vibrational features inferred from FPMD simulations. To this aim one can begin from the consideration that glassy $\mathrm{GeTe}_{4}$ is a semiconductor [19] with thermal transport dominated by the ionic contribution. The eigenvalues and eigenvectors are obtained in the standard way by diagonalization of the Hessian. The vibrational density of states calculated for model B is given in Fig. 2. We obtain a sharp peak, the so-called boson peak, also evidence in inset, observed systematically in disordered materials. Recently this peak has been attributed [25] to a competition between elastic mode propagation and diffusive damping (propagons and diffusons respectively in the taxonomy of Ref. 5). The location of the peak at 120 $\mathrm{cm}^{-1}$ corresponds to the experimental value attributed to the corner sharing tetrahedral units in $\mathrm{GeTe}_{4}$ [26-28].

We have employed the vibrational eigenvectors to quantify the localization of the vibrational modes by calculating the participation ratio $p_{n}$ (see Fig. 3 for model B) defined as [29, 30]:

$$
p_{n}\left(\omega_{n}\right)=\left(\sum_{i=1}^{N}\left\|\overrightarrow{e_{i}}\left(\omega_{n}\right)\right\|^{2}\right)^{2} /\left(N \sum_{i=1}^{N}\left(\left\|\overrightarrow{e_{i}}\left(\omega_{n}\right)\right\|^{2}\right)^{2}\right)
$$

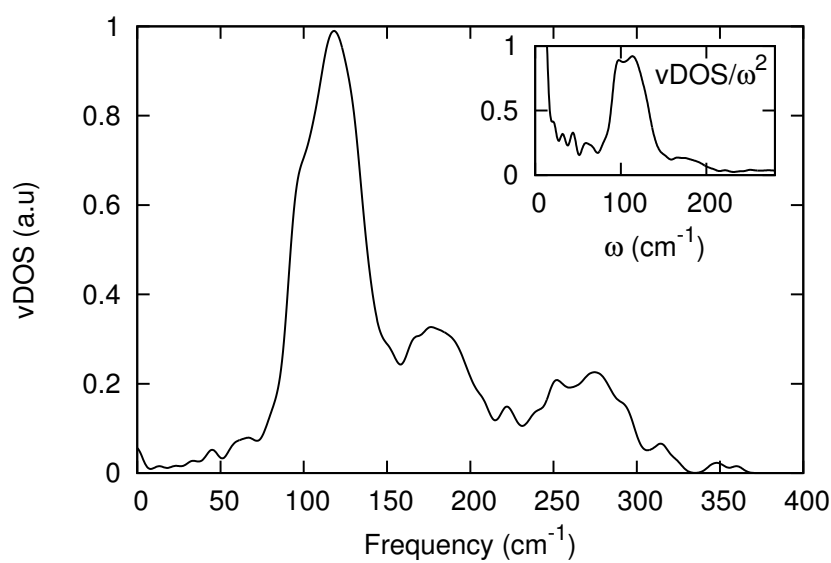

FIG. 2: Vibrational density of states (vDOS). A Gaussian broadening of $5 \mathrm{~cm}^{-1}$ has been employed. In inset, vDOS divided by the frequency square $\omega^{2}$.

for a mode of eigenvalue $\omega_{n}$ and eigenvector $\overrightarrow{e_{i}}\left(\omega_{n}\right)$. The participation ratio of a fully delocalized vibrational mode is equal to 1 , while at the extreme opposite (localization on a single atom), it drops to $1 / N$. The shape of

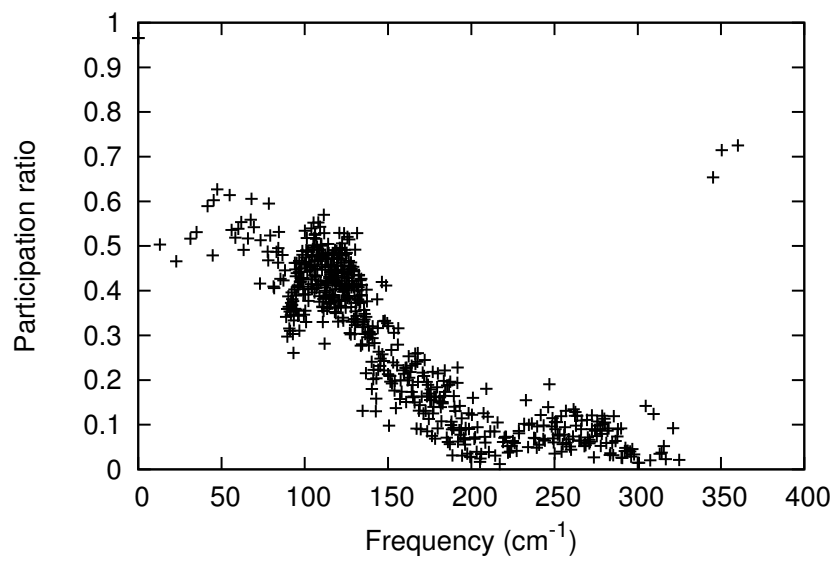

FIG. 3: Participation ratio of the vibrational modes. Model B.

the participation ratio is remindful of what observed in other glasses 29,3133 . The modes at low frequency, i.e. lower than the boson peak frequency, involve as many as $50 \%$ of the atoms while more localized vibrations (defined as locons in Ref. 5) appear around 200 and 300 $\mathrm{cm}^{-1}$. Fig. 4 exemplifies the difference between an extended and a localized mode. The number of atoms significantly involved in vibrational modes is considerably higher at $120 \mathrm{~cm}^{-1}$ than at $300 \mathrm{~cm}^{-1}$. We recall that the modes localized on a few atoms, the locons, do not contribute to the heat transport. 

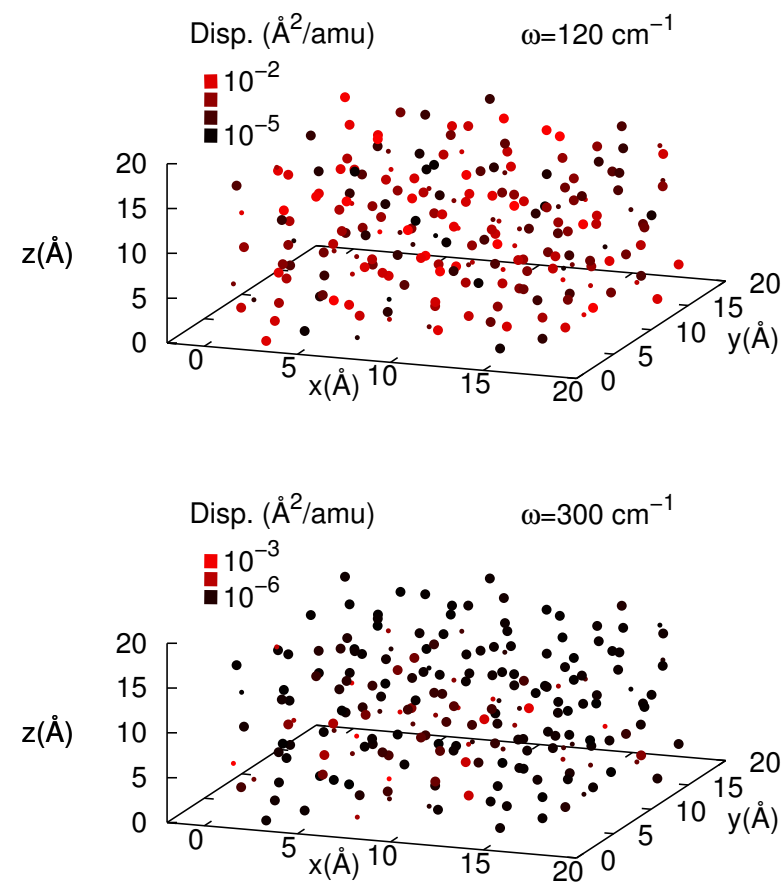

FIG. 4: Atomic displacements for an extended mode at 120 $\mathrm{cm}^{-1}$ and a localized mode at $300 \mathrm{~cm}^{-1}$. Large dots represent Te atoms, small dots are Ge atoms. The color code, from black to light red is an expression of the atomic displacement (Disp.) $\left\|\overrightarrow{e_{i}}\right\|^{2} / m_{i}\left(m_{i}\right.$ being the mass of atom $\left.i\right)$. The analysis has been carried out for model B.

\section{B. Thermal conductivity via AEMD: basic foundations and results}

We calculate the thermal conductivity via the approach-to-equilibrium molecular dynamics (AEMD) method 12. Within this technique, a thermal process can be described by relying on a thermal transient and, in particular, its decay time. This leads to a reduction in computational time by at least one order of magnitude when compared to other molecular dynamics approaches, thereby allowing for affordable applications of FPMD [1].

It is instructive to recall the main features of the AEMD method. A given system is divided in two blocks, kept at different temperatures (hot and cold) during a first phase termed phase 1 (see Fig. 1). Due to periodicity, hot and cold blocks are infinitely propagated in space. At the end of phase 1 one releases the temperature difference between the blocks, leading to a transient regime (phase 2) characterized by the decrease (increase) in temperature of the hot (cold) block. The underlying conceptual framework views the transient regime of phase 2 as the transient regime of the heat equation expressed through a Fourier series, providing a direct access to the determination of the thermal conductivity.

Fig. 5 shows that the process consisting of phases 1 and phase 2 can be readily followed in time by FPMD. The target temperature here is $T_{\mathrm{t}}=130 \mathrm{~K}$, corresponding to the minimum of the mean free paths of heat carriers as indicated by Zhang et al. 34 via their measurements. Size effects are unavoidable in the AEMD methodology 35]. They manifest themselves when the heat carrier mean free paths are larger than the computational cell. For this reason, it is preferable to work at a temperature minimizing the mean free paths. Also, the choice of a temperature larger than the Debye temperature prevents from the consideration of quantum effects. For glassy $\mathrm{GeTe}_{4}$, Zhang et al 34 measured a Debye temperature of $110 \mathrm{~K}$. Taken altogether, the above considerations led us to choose a target temperature of $130 \mathrm{~K}$.

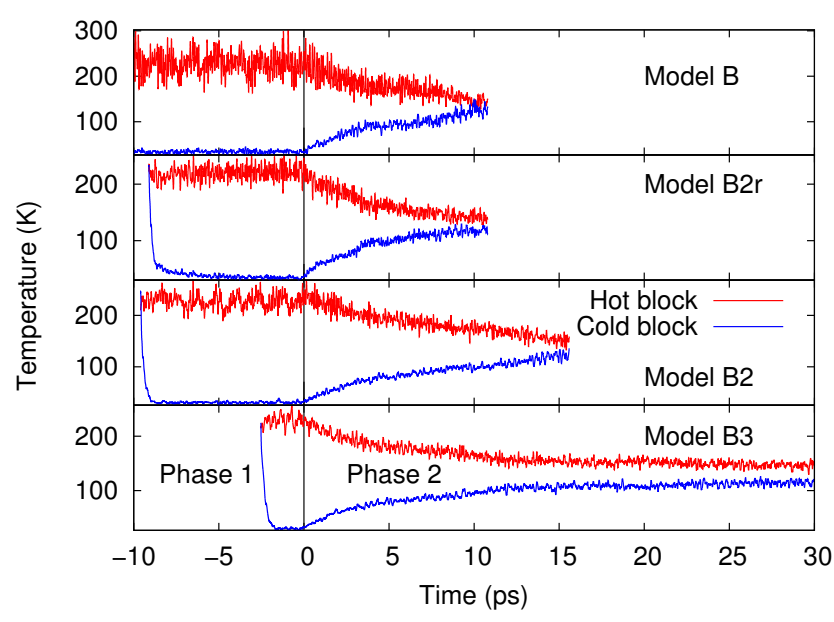

FIG. 5: Temperatures in the hot (red line) and cold (blue line) block during the 2 phases of AEMD, and in models B, B2, B2r and B3.

First we equilibrated our full computational sample at the temperature $T=230 \mathrm{~K}$ (see Fig. 5). We then form the two blocks of Fig. 1 by applying a local thermostat at $230 \mathrm{~K}$ to the atoms of the hot block and at $30 \mathrm{~K}$ to the atoms of the cold block. Upon application of these two local thermostats, a temperature difference of $\Delta T_{0}=200$ $\mathrm{K}$ is obtained in less than 1 ps (see Fig. 5). Typically, the thermostats are applied during $10 \mathrm{ps,} \mathrm{although} \mathrm{a} \mathrm{du-}$ ration as long as $80 \mathrm{ps}$ have been tested in a previous work [11. For model B3, phase 1 lasts only 2.5 ps, due to the higher computational cost of FPMD for a system containing 645 atoms. The duration of phase 1 does not have a significant impact on the final result provided the asymptotic decay time is carefully extracted from phase 2 by discarding a short initial transient, occurring in the thermal transition between phase 1 (canonical ensemble, two thermostats) and phase 2 (microcanonical ensemble).

Focusing on phase 2, we first recall that AEMD is reminiscent of the heat conduction problem for a ring [36. The solution $T(t, z)$ along the transport direction $z$ is a 
Fourier series 12 :

$T(t, z)=T_{t}+\sum_{m=0}^{\infty} \frac{2 \Delta T_{0}}{(2 m+1) \pi} \sin \left(\frac{2 \pi(2 m+1)}{L} z\right) e^{-(2 m+1)^{2} t /}$

with the decay time $\tau$ related to the thermal diffusivity $D$ as follows

$$
\frac{1}{\tau}=\frac{4 \pi^{2}}{L^{2}} D
$$

The diffusivity of a material of thermal conductivity $\kappa$, number density $\rho$ and heat capacity $C$ is equal to $D=$ $\kappa /(C \rho)$. By considering the dominant contribution of the Fourier series $(m=0)$ (a decaying sine) one obtains

$$
T(t, z)-T_{\mathrm{t}} \propto \Delta T_{0} \sin \left(\frac{2 \pi z}{L}\right) e^{-t / \tau}
$$

The Fourier series (Eq. 2) can be applied to express the temperature difference between the two blocks

$$
\Delta T(t)=\frac{2}{L} \int_{0}^{L / 2} T(t, z) d z-\frac{2}{L} \int_{L / 2}^{L} T(t, z) d z
$$

under the form featuring the dominant contribution

$$
\Delta T(t) \propto \Delta T_{0} e^{-t / \tau} .
$$

Therefore, provided we can demonstrate that the temperature profile and the temperature difference $\Delta T$ do obey Eqs. 4 and 6, the decay time $\tau$ of the thermal transient gives access to the thermal conductivity $\kappa$ via:

$$
\kappa=\frac{L^{2}}{4 \pi^{2}} \frac{C \cdot \rho}{\tau} .
$$

To ensure full consistency between the underlying theory of thermal transport and the AEMD implementation we check the form of the temperature profile and the time behavior of the temperature difference. These quantities $(T(z)$ and $\Delta T(t))$ are given respectively in Figs. 6 and 7 during phase 2 for models B, B2, B2r and B3, and an initial temperature difference of $200 \mathrm{~K}$. The temperature profiles have a sine form, as shown by the sine functions superimposed on the AEMD results. Concerning Fig. 6 we adopted the same range for the $z$ range regardless of their different longitudinal extension, by replicating the temperature profiles of models B, B2r and B2 as allowed by the periodic boundaries conditions.

The AEMD temperature difference $\Delta T(t)$ evolves to a very good extend according to an exponential decay (Fig. 7). The exponential fit allows determining the decay time $\tau$ for each system. In model B3 one observes a short transient to be attributed to the short duration of phase 1 (2.5 ps see Fig. 5). This does not prevent either from the observation of an asymptotic exponential regime on 25 ps for an amplitude of $100 \mathrm{~K}$, or from determining the relevant decay time $\tau$.

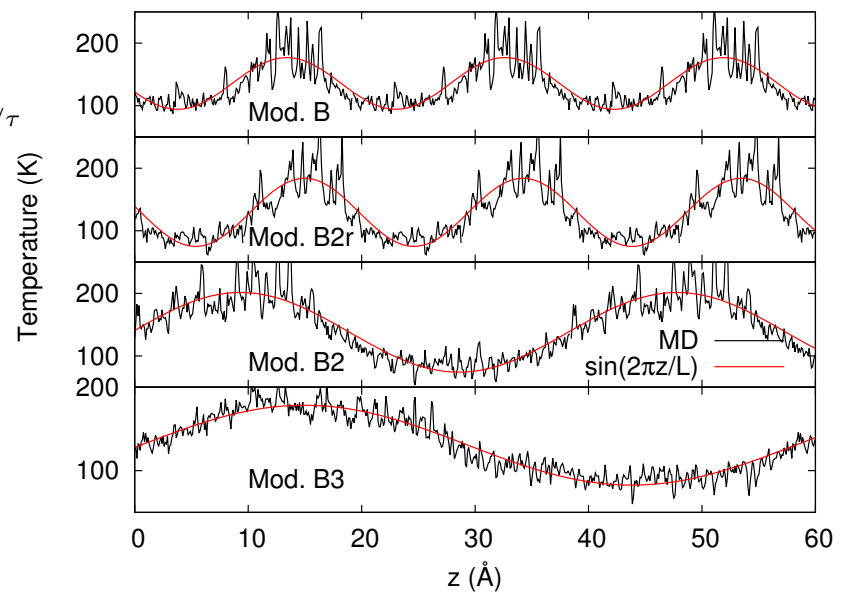

FIG. 6: Temperature profile along the transport direction, averaged during phase 2 of AEMD. The black lines are the MD results. The red lines are sine functions (Eq. 4).

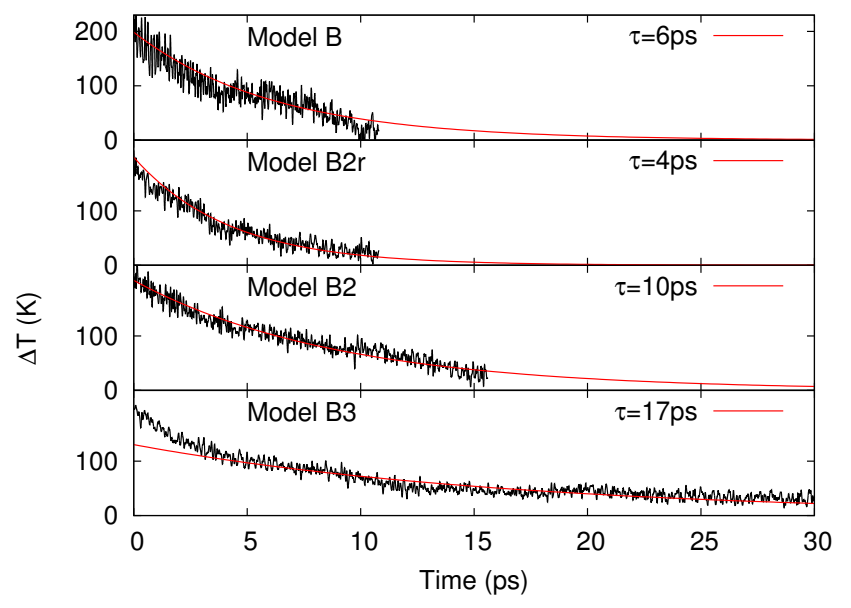

FIG. 7: Evolution with time of the temperature difference between the hot and the cold blocks during phase 2 of AEMD. The black lines are the MD results. The red lines are exponential decays (Eq. 6), the value of $\tau$ being reported in the legend.

The last ingredient to obtain the thermal conductivity from Eq. 7 is the heat capacity $C$ that we calculated from the variation of the total energy versus temperature (Fig. 8). The curve is linear and its slope gives a heat capacity of $C=1.024 C_{0}$, the Dulong and Petit value being $C_{0}=3 N k_{\mathrm{B}}$. When repeating the calculation for models B2 and B3 we did not observe any noticeable size effects. The average value, used in the following, is $C=(1.025 \pm 0.003) C_{0}$.

Our results for the thermal conductivity are presented in Fig. 9. In particular, for model B, we tested two values for the initial difference in temperature $\Delta T_{0}$ and two values for the duration of phase 1 . It was found that an initial $\Delta T_{0}$ of $200 \mathrm{~K}$ contributes to reducing the statistical uncertainty, while the length of phase 1 duration has a negligible effect [11, 13. Regarding the dimension trans- 


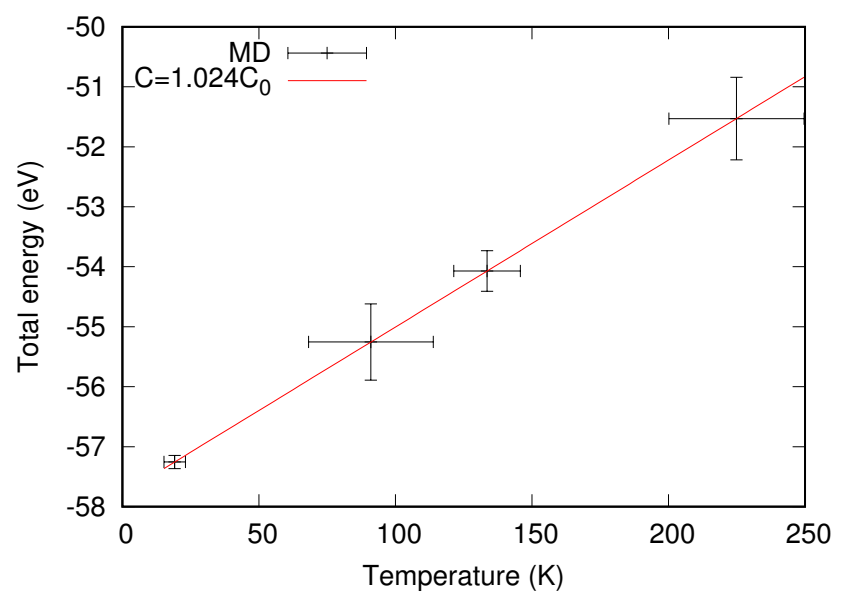

FIG. 8: Total energy versus temperature and fit to obtain the heat capacity. Model B.

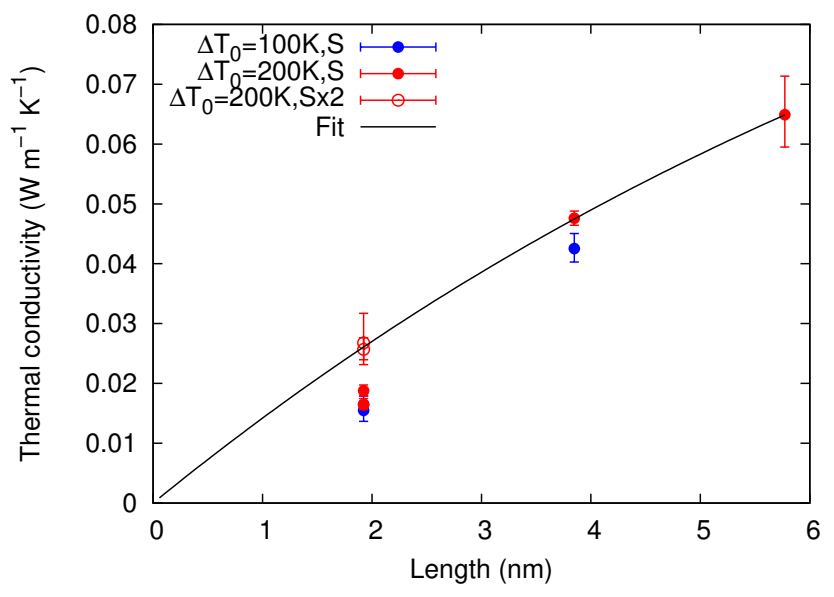

FIG. 9: Thermal conductivity versus length. Dots of different colors are related to the temperature difference $\Delta T_{0}$ imposed during phase 1 (red for $200 \mathrm{~K}$ and blue for $100 \mathrm{~K}$ ). The full circles are result for the small cross section termed $\mathrm{S}$ (models B, B2 and B3). The open circle is for model B2r, with a section $\mathrm{Sx} 2$ of double size. The black line is a fit following Alvarez' equation 37] with $\kappa_{\infty}=0.15 \mathrm{~W} \mathrm{~K}^{-1} \mathrm{~m}^{-1}$ and $l=3.2$ $\mathrm{nm}$.

verse to the heat flux, the results for model B2r, larger than for model B, indicate that this parameter has an impact on the results, at least for small sizes, thereby confirming the validity of our global approach that consisted in taking systems of four distinct geometries.

A dependence on the size of the system through the box length $L$ (or, equivalently, on the periodicity of the blocks thermally constrained to be hot or cold) is noticeable in Fig. 9 by confirming preliminary results of Ref. 13 for the range [0-4] $\mathrm{nm}$. Interestingly, the thermal conductivity goes on increasing even when the box length in the direction of heat transport is $6 \mathrm{~nm}$ (system B3). This can be interpreted by invoking a propagation of heat carriers (propagons) up to distances of $6 \mathrm{~nm}$ at least. Propagons are identified both in calculations and experiments via a dependence of the thermal conductivity on the sample size or via a thermal conductivity versus mean free paths spectroscopy technique [7]. In a recent review of thermal transport in amorphous materials, Winger et al. [8] reported that propagons have been identified in amorphous silicon (a-Si) but no variation of the thermal conductivity with size has been detected in oxide glasses, such $\mathrm{SiO}_{2}$, $\mathrm{HfO}_{2}, \mathrm{Al}_{2} \mathrm{O}_{3}$ and $\mathrm{TiO}_{2}$. To the best of our knowledge, no evidence of propagons has ever been reported on chalcogenide glasses. Here we prove that propagons, although with mean free paths drastically lower than in a-Si, do exist also in glassy chalcogenides as g- $\mathrm{GeTe}_{4}$. One might even conjecture that this could be the case for the oxide glasses since so far no thermal characterization below 5 $\mathrm{nm}$ has been achieved while this range of dimensions is readily accessible to FPMD.

The idea of resorting to a system more extended than B3 in direction of heat transport is not necessarily tractable in view of the associated computational cost. One can instead take advantage of the collected data for B2 and B3 in order to extrapolate our results using the equation developed by Alvarez and Jou 37. This relationship describes the ballistic to diffusive regime inherent in non-local effects observed at small sizes in AEMD 38]:

$$
\kappa(L)=\kappa_{\infty} \frac{L^{2}}{2 \pi^{2} l^{2}}\left[\sqrt{1+4\left(\frac{\pi l}{L}\right)^{2}}-1\right],
$$

where $\kappa_{\infty}$ is the thermal conductivity at saturation and $l$ is a mean free path. The fit is reported as a black line in Fig. 9. The thermal conductivity converges to a value of $0.15 \mathrm{~W} \mathrm{~K}^{-1} \mathrm{~m}^{-1}$ with a statistical uncertainty of 0.01 $\mathrm{W} \mathrm{K} \mathrm{K}^{-1} \mathrm{~m}^{-1}$. The thermal conductivity of $\mathrm{g}-\mathrm{GeTe}_{4}$ has been measured by Zhang et al. 34 using a parallel temperature conductance (PTC) technique. They obtained a value of $0.14 \mathrm{~W} \mathrm{~K}^{-1} \mathrm{~m}^{-1}$ at $130 \mathrm{~K}$. We conclude that the extrapolation to infinite (macroscopic) sizes of our calculations is in excellent agreement with this measurement.

\section{VALIDITY OF PHENOMENOLOGICAL MODELS}

The availability of the vibrational spectrum, heat capacity and thermal conductivity of $\mathrm{g}-\mathrm{GeTe}_{4}$ allows establishing the predictive power of phenomenological models for glasses. This issue is of paramount importance both from the fundamental and applied point of view since fully reliable models are needed to guide the design of thermoelectric or memory devices.

In what follows, three models will be under scrutiny. The first is based on the gas kinetic equation, the second is the so-called "minimum thermal conductivity" model by Cahill and Pohl [39, 40] and the third is due by Agne et al. 41. The first two models assume that thermal 
transport is due to propagative heat carriers, while the third one was developed under the hypothesis of the existence of diffusons.

Focusing on the first model, the gas kinetic equation relates the thermal conductivity to three quantities: the volumetric specific heat capacity $c=C / V$ of the excitations providing the thermal transport, the sound velocity $v$ at which they propagate and the mean free path between scattering events $l$ :

$$
\kappa_{\mathrm{Kin}}=\frac{1}{3} c v l
$$

The value of $l$ obtained from our $\kappa(L)$ results is employed, i.e. $l=3.2 \mathrm{~nm}$ (Fig. 9). The speed of sound can be obtained from our FPMD calculations since the alternation of hot and cold blocks inherent in phase 1 of AEMD leads to a deformation wave that superimposes to the temperature profile during phase 2, as shown in Fig. 10. The

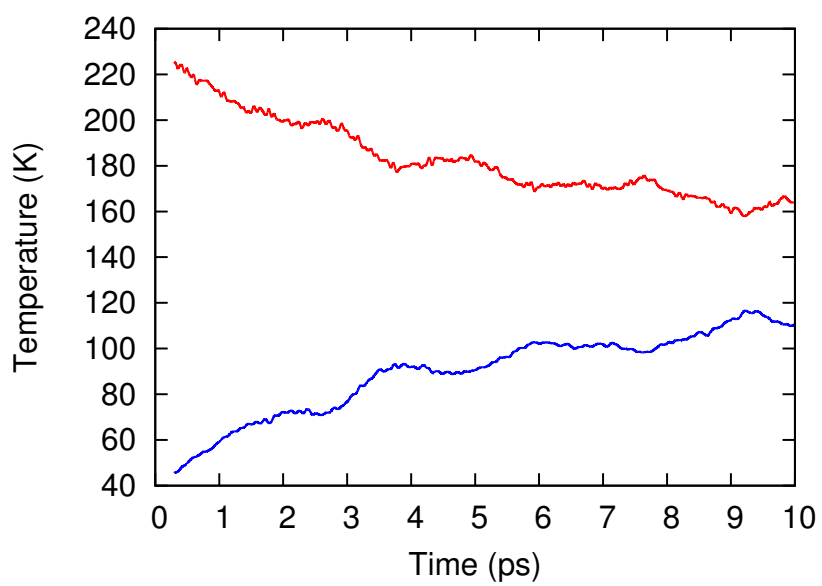

FIG. 10: Oscillations in the temperature evolution of the hot and cold blocks. The statistical noise is attenuated by applying a sliding average on intervals of $600 \mathrm{fs}$.

temperature curves have been numerically derived in Fig. 11 so as to obtain the position of the minima in the hot temperature curve and maxima in the cold one. This procedure has been repeated as a function of the cell length (Fig. 12) and leads to a sound velocity $v=1 \mathrm{~nm} / \mathrm{ps}$. Therefore, the estimate of the thermal conductivity from the gas kinetic theory is $\kappa_{\text {Kin }}=1.36 \mathrm{~W} \mathrm{~K}^{-1} \mathrm{~m}^{-1}$, nearly ten times larger than the result of our calculation.

The model by Cahill and Pohl [39, 40] views propagation of heat as due to coupling of harmonic oscillators. In this model the oscillating entities are regions of size equal to half the vibration wavelength. The analytical expression resulting from this hypothesis is:

$$
\kappa_{\mathrm{CP}}=\left(\frac{\pi}{6}\right)^{\frac{1}{3}} k_{\mathrm{B}} \rho^{\frac{2}{3}} \sum_{i} v_{i}\left(\frac{T}{\Theta_{i}}\right)^{2} \int_{0}^{\frac{\Theta_{i}}{T}} \frac{x^{3} e^{x}}{\left(e^{x}-1\right)^{2}} d x
$$

where the sum is taken over the longitudinal and two transverse sound modes and $\Theta_{i}=v_{i}\left(\hbar / k_{\mathrm{B}}\right)\left(6 \pi^{2} \rho\right)^{\frac{1}{3}}$.

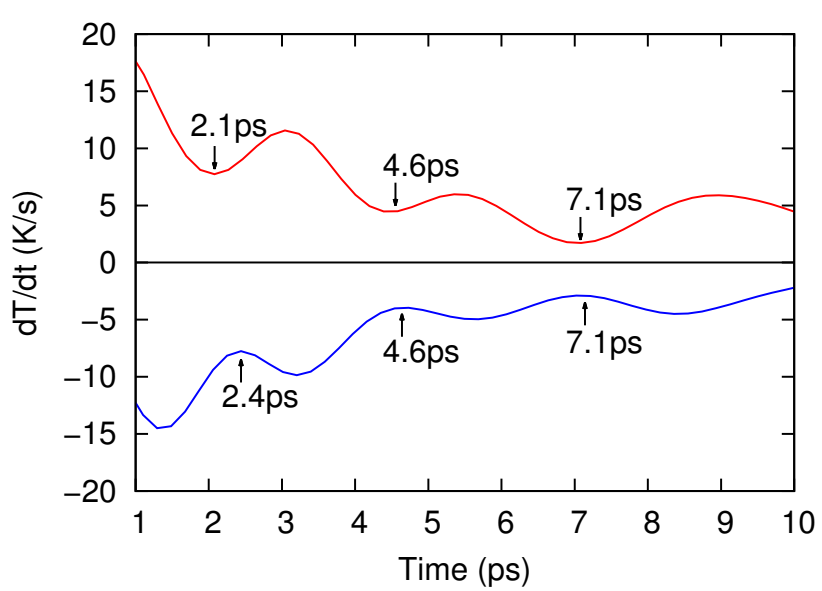

FIG. 11: Derivatives of temperatures shown in Fig. 10

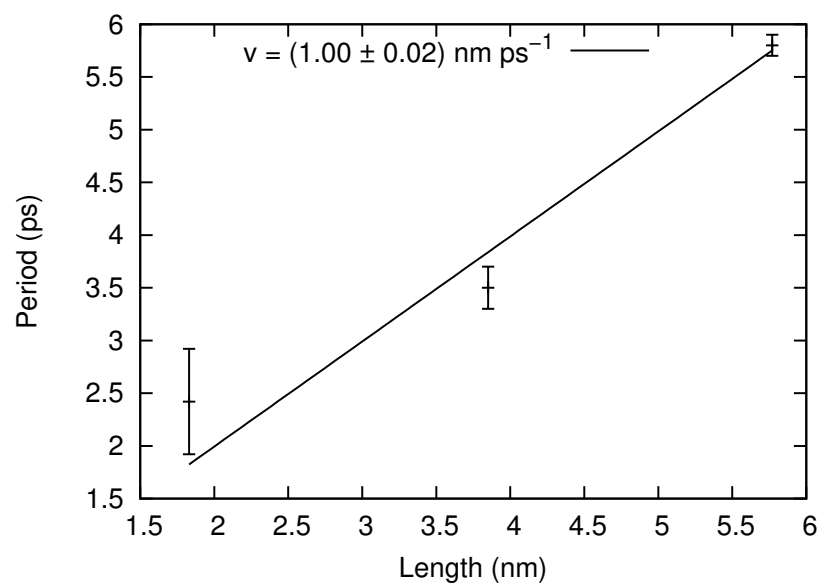

FIG. 12: Sound velocity extracted from the oscillations in the temperature evolution with time.

Here we consider the same sound velocity for the three modes, and proceed to a numerical integration giving a value of $\kappa_{\mathrm{CP}}=0.16 \mathrm{~W} \mathrm{~K}^{-1} \mathrm{~m}^{-1}$, in very good agreement with the value we calculated.

Finally, let us consider the model proposed by Agne and co-workers 41 fully based on diffusons, i. e. without involving the notion of mean free path. The diffusivity of coupled harmonic oscillators is calculated assuming an isotropic material, leading to a thermal conductivity that reads

$$
\kappa_{\text {Diff }}=\frac{1}{\pi} n^{\frac{1}{3}} k_{\mathrm{B}} \omega_{\text {avg }}
$$

For $\omega_{\text {avg }}$ we can take the average oscillator frequency that we calculated from the vibrational density of states $(\approx$ $\left.140 \mathrm{~cm}^{-1}\right)$. The resulting thermal conductivity is equal to $\kappa_{\text {Diff }}=0.06 \mathrm{~W} \mathrm{~K}^{-1} \mathrm{~m}^{-1}$. As expected, this estimate is lower than the thermal conductivity calculated by FPMD since it does not account for propagative modes.

The values obtained with the above models are reported in Fig. 13 together with our FPMD calculation 


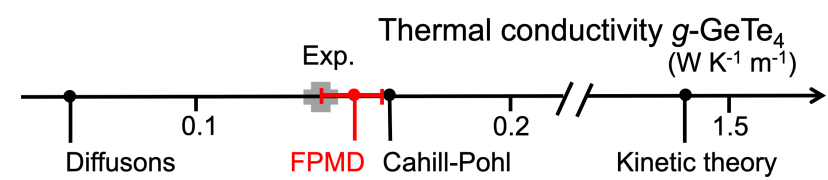

FIG. 13: Thermal conductivities as obtained via the phenomenological models considered in our analysis (diffusons 41, minimum thermal conductivity by Cahill and Pohl [39, 40] and gas kinetic theory). The models have been enriched by FPMD calculated values of specific thermal properties (see text). A comparison is carried out with our FPMD calculation and with experimental measurements 34.

and the experimental data. Despite its qualitative foundations and purposes, it appears that the minimum thermal conductivity model by Cahill and Pohl is the most realistic one after inclusion (in the expression of the thermal conductivity) of a property (the sound velocity) evaluated by FPMD calculations.

\section{CONCLUSIONS}

We reported a first-principles molecular dynamics study (supplemented by a DFT-based analysis of vibrational modes) of the thermal properties of the $\mathrm{GeTe}_{4}$ glass. Glassy $\mathrm{GeTe}_{4}$ is close in composition to phasechange chalcogenides at the core of modern non-volatile memory devices. The vibrational spectrum revealed the existence of localized modes. We rationalized thermal transport by calculating the thermal conductivity via the approach-to-equilibrium methodology applied to four distinct systems differing by the size and/or the shape of the computational box. Striking evidence is collected on the occurrence of propagative modes extending up to $6 \mathrm{~nm}$. By asymptotic extrapolation of our calculations we obtained a thermal conductivity equal to $0.15 \pm 0.01 \mathrm{~W}$
$\mathrm{K}^{-1} \mathrm{~m}^{-1}$ at $130 \mathrm{~K}$ for the bulk, in excellent agreement with the experimental value. As a final piece of information, we have revisited phenomenological models of heat transport by including in their analytical expression specific quantities calculated via FPMD. The assumption of a diffusion-mediated transport made by Agne et al.41. leads to a sever underestimate of the thermal conductivity, in line with the importance of propagative modes not included in this model. On the other hand, the minimum thermal conductivity approach by Cahill and Pohl 39, 40, enriched by FPMD calculations of the sound velocity, agrees well with our extrapolated value.

This work is a breakthrough toward precise understanding of the heat transport in disordered materials, since it highlights the existence of propagative modes well beyond the first-neighbor distances in a system other than disordered silicon. The mean free paths of the heat carriers in the present chalcogenide glass falls in the nanometer range and yet they are too small to be accessible to measurements, currently hampering any experimental confirmation. Overall, it appears that the combined use of FPMD and AEMD is well suited to describe heat transport and obtain realistic values of the thermal conductivity, due to the treatment of transients containing invaluable information on thermal transport at the nanoscale.

\section{ACKNOWLEDGEMENTS}

Financial support of the French ANR via the project n. ANR-17-CE09-0039-02 "SIRENA" is gratefully acknowledged. Calculations were performed by using resources from GENCI (Grand Equipement National de Calcul Intensif) (Grants No. x2016095071, A0030910296, A0040905071, A0050910296), Pôle HPC Equip@Meso of the University of Strasbourg, and clustphy2 at the IEMN.
[1] M. Wuttig, H. Bhaskaran, and T. Taubner, Nature Photonics 11, 465 (2017).

[2] T. D. Lee and A. U. Ebong, Renewable and Sustainable Energy Reviews 70, 1286 (2017).

[3] D. Banerjee, Ö. Vallin, K. M. Samani, S. Majee, S.-L. Zhang, J. Liu, and Z.-B. Zhang, Nano Energy 44, 89 (2018).

[4] P. Noé, C. Vallée, F. Hippert, F. Fillot, and J.-Y. Raty, Semicond. Sci. Technol. 33, 013002 (2017).

[5] P. B. Allen, J. L. Feldman, J. Fabian, and F. Wooten, Philos. Mag. B 79, 1715 (1999)

[6] Y. He, D. Donadio, and G. Galli, Appl. Phys. Lett. 98, 144101 (2011).

[7] K. T. Regner, D. P. Sellan, Z. Su, C. H. Amon, A. J. McGaughey, and J. A. Malen, Nature Communications 4, 1640 (2013).

[8] M. C. Wingert, J. Zheng, S. Kwon, and R. Chen, Semi- cond. Sci. Technol. 31, 113003 (2016).

[9] R. Zwanzig, Annu. Rev. Phys. Chem. 16, 67 (1965).

[10] P. K. Schelling, S. R. Phillpot, and P. Keblinski, Phys. Rev. B 65, 144306 (2002).

[11] A. Bouzid, H. Zaoui, P. L. Palla, G. Ori, M. Boero, C. Massobrio, F. Cleri, and E. Lampin, Phys. Chem. Chem. Phys. 19, 9729 (2017).

[12] E. Lampin, P. L. Palla, P.-A. Francioso, and F. Cleri, Journal of Applied Physics 114, 033525 (2013).

[13] E. Martin, P. L. Palla, F. Cleri, A. Bouzid, G. Ori, S. Le Roux, M. Boero, and C. Massobrio, Journal of NonCrystalline Solids 498, 190 (2018).

[14] R. Car and M. Parrinello, Phys. Rev. Lett. 55, 2471 (1985).

[15] A. D. Becke, Phys. Rev. A 38, 3098 (1988).

[16] C. Lee, W. Yang, and R. G. Parr, Phys. Rev. B 37, 785 (1988). 
[17] J. P. Perdew, K. Burke, and M. Ernzerhof, Phys. Rev. Lett. 77, 3865 (1996)

[18] N. Troullier and J. L. Martins, Phys. Rev. B 43, 1993 (1991).

[19] A. Bouzid, C. Massobrio, M. Boero, G. Ori, K. Sykina, and E. Furet, Phys. Rev. B 92, 134208 (2015).

[20] S. Grimme, J. Comput. Chem. 27, 1787 (2006).

[21] S. Nosé, Mol. Phys. 52, 255 (1984).

[22] W. G. Hoover, Phys. Rev. A 31, 1695 (1985).

[23] P. E. Blöchl and M. Parrinello, Phys. Rev. B 45, 9413 (1992).

[24] D. Dubbeldam, S. Calero, and T. J. H. Vlugt, Mol Simulat 44, 653 (2018).

[25] M. Baggioli and A. Zaccone, Phys. Rev. Lett. 122, 145501 (2019).

[26] K. S. Andrikopoulos, S. N. Yannopoulos, A. V. Kolobov, P. Fons, and J. Tominaga, Journal of Physics and Chemistry of Solids 68, 1074 (2007).

[27] M. Upadhyay, S. Murugavel, M. Anbarasu, and T. R. Ravindran, Journal of Applied Physics 110, 083711 (2011).

[28] K. Makino, J. Tominaga, and M. Hase, Opt. Express, OE 19, 1260 (2011).

[29] R. J. Bell, P. Dean, and D. C. Hibbins-Butler, J. Phys. C: Solid State Phys. 3, 2111 (1970).

[30] B. B. Laird and H. R. Schober, Phys. Rev. Lett. 66, 636 (1991).
[31] S. N. Taraskin and S. R. Elliott, Phys. Rev. B 56, 8605 (1997).

[32] N. Zotov, I. Ebbsjö, D. Timpel, and H. Keppler, Phys. Rev. B 60, 6383 (1999).

[33] A. Tanguy, JOM 67, 1832 (2015).

[34] S.-N. Zhang, J. He, T.-J. Zhu, X.-B. Zhao, and T. M. Tritt, Journal of Non-Crystalline Solids 355, 79 (2009).

[35] H. Zaoui, P. L. Palla, F. Cleri, and E. Lampin, Phys. Rev. B 94, 054304 (2016).

[36] A. Sommerfeld, Lectures on Theoretical Physics, Partial Differential Equations in Physics, Vol.6 (Academic Press, 1949)

[37] F. X. Alvarez and D. Jou, Appl. Phys. Lett. 90, 083109 (2007).

[38] P. L. Palla, S. Zampa, E. Martin, and F. Cleri, International Journal of Heat and Mass Transfer 131, 932 (2019).

[39] D. G. Cahill and R. O. Pohl, Annu. Rev. Phys. Chem. 39, 93 (1988).

[40] D. G. Cahill and R. O. Pohl, Solid State Communications 70, 927 (1989).

[41] M. T. Agne, R. Hanus, and G. J. Snyder, Energy Environ. Sci. 11, 609 (2018).

[42] See http://www.cpmd.org, copyright 2000-2017 jointly by IBM Corporation and by Max Planck Institute, Stuttgart 